\title{
Using Scan Statistic to Detect Heroine Overdose Clusters with Hospital Emergency Room Visit Data
}

\author{
Jianhua Chen*, Hwa-Gan Chang, Mark Hammer, Nicole D’Anna and Kitty Gelberg
}

New York State Department of Health, Albany, NY, USA

\section{Objective}

To utilize syndromic surveillance data timely detecting herion overdose outbreaks in the community.

\section{Introduction}

Early detection of heroin overdose clusters is important in the current battle against the opioid crisis to effectively implement prevention and control measures. The New York State syndromic surveillance system collects hospital emergency department (ED) visit data, including visit time, chief complaint, and patient zip code. This data can be used to timely identify potential heroin overdose outbreaks by detecting spatial-temporal case clusters with scan statistic.

\section{Methods}

Heroin overdose cases (Heroin OD) were identified from ED visits by searching Heroin_OD key terms in the chief complaints. Then the space-time permutation model (using the SaTScan package) was applied to detect clusters of Heroin_OD. ED visit date served as the time variable and the case residential zip code was the spatial coordinate variable for the SaTScan analysis. A SAS program was developed to carry out the prospective scan statistics analysis weekly and produces reports of detected clusters in table and map format. Cluster detection parameters were set to detect heroin overdose aggregation in maximum geographic radium of 20 kilometer $(\mathrm{km})$ and maximum time span up of 21 days at the P-value $<=0.05$. Chief complaints within the clusters are reviewed to ensure accuracy of detection. Messages have been developed and are shared with community members including law enforcement and public health identifying the cluster and offering suggestions of activities that can occur at the local level to identify and address the cause of the cluster, as well as to reduce potential harm. This includes the 23 syringe exchange programs (SEPs) regulated by the New York State Department of Health.

\section{Results}

Using ED visit data from $138 \mathrm{NY}$ upstate hospitals, a total of 12 Heroin_OD clusters were detected by the SaTScan analysis during the period of $9 / 1 / 2016$ through $9 / 17 / 2017$. There were 845 cases identified. The average age was 35 years and ranged from 7 to 95 years. $69 \%$ of the cases were on the 20 to 39 age group and $66 \%$ were among males. A cluster was identified earlier 2017 in Suffolk County, and the local SEP was alerted. This encouraged communication between partners within the alerted county which ultimately resulted in identifying the substance endangering people who used drugs in the area. It also helped public health to partner with public safety, ensuring that the availability of the substance was interrupted.

\section{Conclusions}

As the space-time permutation scan statistic only requires disease counts, event date and disease location, the method can be easily implemented for detecting disease outbreaks using data routinely collected from disease surveillance systems. The current study showed that scan statistic is a useful tool for identifying clusters of non-fatal overdoses from specific drugs. This method also returns important information to assist outbreak investigations, such as geographic location and time-span of the potential outbreaks.

Keywords

heroin overdose; cluster detection; scan statistics

\section{*Jianhua Chen}

E-mail: jianhua.chen@health.ny.gov 